\title{
Efficacy and superiority between endoscopic retrograde cholangiopancreatography and percutaneous transhepatic biliary drainage as first line intervention of biliary complication after liver transplantation
}

\author{
Minseob KIM, Suk Kyun HONG*
}

Department of Surgery, Seoul National University Hospital, Seoul, Korea

Introduction: Biliary complications after liver transplantation (LT) are the most common complications and associated with morbidity and mortality. There has not been a uniform conclusion published on the superiority of the two types of intervention as the first trial. Therefore, we compared the efficacy of endoscopic retrograde cholangiopancreatography (ERCP) and percutaneous transhepatic biliary drainage (PTBD) as a first-line treatment of post-LT biliary problems.

Methods: From January 2013 to December 2016, 565 patients underwent LT in Seoul National University Hospital. Medical records of recipients who were diagnosed with biliary complications after LT retrospectively reviewed. Long-term follow-up was evaluated using cholangiogram, computed tomography (CT) scan, and laboratory parameters.

Results: Among 565 LT patients, 85 patients (15.0\%) were treated by intervention including ERCP and PTBD with diagnosis of biliary complications. Successful intervention on the first attempt was achieved in 36 of 60 patients (60.0\%) with ERCP, and 19 of 25 patients (76.0\%) with PTBD, respectively $(p=0.16)$. We also classified the groups based on the location of the biliary complications and compared the success rate; one with anterior bile duct problems $(a-B D, n=29)$ and another with posterior bile duct $(p-B D, n=14)$. In a-BD, there was no difference in the intervention success rate of PTBD and ERCP (25\% vs. 24\%, $p=0.692)$. However, in p-BD, PTBD success rate was significantly better than ERCP ( $75 \%$ vs. $18 \%, p=0.002)$.

Conclusions: PTBD could be considered as more effective procedure for termination of treatment in patients with post-LT biliary complications, especially posterior posterior duct problems. 